DR. MARTA MELIS (Orcid ID : 0000-0001-6037-4125)

DR. MARGHERITA FABBRI (Orcid ID : 0000-0001-7250-592X)

DR. MAURIZIO ZIBETTI (Orcid ID : 0000-0002-2939-343X)

DR. GIOVANNI COSSU (Orcid ID : 0000-0002-9129-2584)

Article type : Original Article

\title{
GUT MICROBIOTA AND METABOLOME DISTINCTIVE FEATURES IN PARKINSON DISEASE: FOCUS ON LEVODOPA AND LEVODOPA CARBIDOPA INTRAJEJUNAL GEL.
}

Marta Melis, MD,${ }^{1 *}$ Sarah Vascellari, ${ }^{2 * \wedge}$ Maria Laura Santoru, ${ }^{3}$ Valentina Oppo, ${ }^{4}$ Margherita Fabbri, ${ }^{5}$ Marianna Sarchioto, ${ }^{6}$ Daniela Murgia, ${ }^{4}$ Maurizio Zibetti, ${ }^{5}$ Leonardo Lopiano, ${ }^{5}$ Alessandra Serra, ${ }^{2}$ Vanessa Palmas, ${ }^{2}$ Silvia Pisanu, ${ }^{2}$ Daniela Perra, ${ }^{2}$ Veronica Madau, ${ }^{2}$ Roberto Cusano, ${ }^{7}$ Paolo Uva, ${ }^{7}$ Alessandra Mereu, ${ }^{8}$ Paolo Contu, ${ }^{8}$ Micaela Morelli, ${ }^{9}$ Luigi Atzori, ${ }^{3}$ Maurizio Melis, ${ }^{4}$ Aldo Manzin, $\mathrm{PhD},{ }^{2 * *}$ Giovanni Cossu, $\mathrm{MD},{ }^{4 \wedge}$ **

${ }^{1}$ Department of Neurology AOU Policlinico Monserrato, Cagliari, Italy; ${ }^{2}$ Department of Biomedical Sciences, Section of Microbiology and Virology, University of Cagliari, Italy ${ }^{3}$ Department of Biomedical Sciences, University of Cagliari, Italy; ${ }^{4}$ Department of Neurology AO Brotzu, Cagliari, Italy; ${ }^{5}$ Department of Neurosciences Rita Levi Montalcini, University of Torino, Italy; ${ }^{6}$ Neurosciences Research Centre, Molecular and Clinical Sciences Research Institute, St George's University of London, London, United Kingdom, ${ }^{7}$ CRS4, Science and Technology Park Polaris, Piscina Manna, Pula, Cagliari, Italy; 8 Dipartimento di Scienze Mediche e Sanità Pubblica, Università degli Studi di Cagliari. ${ }^{9}$ Department of Biomedical Sciences, Section of Neuroscience, University of Cagliari, Cagliari, Italy.

This article has been accepted for publication and undergone full peer review but has not been through the copyediting, typesetting, pagination and proofreading process, which may lead to differences between this version and the Version of Record. Please cite this article as doi: 10.1111/ENE.14644

This article is protected by copyright. All rights reserved 


\title{
"Contributed equally to this work
}

\section{** Contributed equally to this work}

Corresponding authors: Giovanni Cossu, Department of Neurology AO Brotzu, Cagliari, Italy, email giovannicossu1@gmail.com, Tel. +3970539668, Sarah Vascellari, Department of Biomedical Sciences, Section of Microbiology and Virology, University of Cagliari, Italy

Department of Neurology, email, svascellari@unica.it Tel. +393332772404

Running Title: LD and mostly LCIG influence the microbiota and host/bacteria metabolism in PD.

\section{Word Count Abstract: 248, Word Count Main Text: 3505}

Keywords: Parkinson, microbiota, Levodopa, LCIG, Duodopa

\begin{abstract}
Background: Recent data suggest that imbalances in the composition of the gut microbiome (GM) could exacerbate the progression of Parkinson's Disease (PD). The effect of Levodopa (LD) has been poorly assessed and those of LD-carbidopa intestinal gel (LCIG) have not been evaluated so far. The aim of this study was to identify the effect of LD and, in particular, LCIG on GM and metabolome.
\end{abstract}

Methods: Faecal DNA samples from 107 patients with clinical diagnosis of PD were analyzed by next-generation-sequencing of V3 and V4 regions of the 16S rRNA gene. PD patients were classified in different groups: patients on LCIG (LCIG-Group) ( $\mathrm{n}=38)$ and on LD (LD-Group) $(n=46)$. We also included a group of patients $(n=23)$ without antiparkinsonian medicaments (Naïve-Group). Faecal metabolic extracts were evaluated by Gas Chromatography Mass Spectrometry (GC-MS).

Results: The multivariate analysis showed a significant higher abundance in the LCIG-Group of Enterobacteriaceae, Escherichia and Serratia compared to LD-Group. Compared to Naïve-Group, the univariate analysis showed a reduction of Blautia, Lachnospirae in LD-Group. Moreover, an 
increase of Proteobacteria, Enterobacteriaceae and a reduction of Firmicutes, Lachnospiraceae and Blautia was found in the LCIG- Group. No significant difference was found in the multivariate analysis of these comparisons. The LD-Group and LCIG-Group were associated to a metabolic profile linked to gut inflammation.

Conclusion: Our results suggest that LD and mostly LCIG might significantly influence the microbiota composition and host/bacteria metabolism acting as stressors in precipitating a specific inflammatory intestinal microenviroment, potentially related to the PD state and progression.

\section{INTRODUCTION}

Besides classical motor symptoms, PD patients frequently show gastrointestinal dysfunctions including impaired gastric emptying, constipation, and defecatory dysfunction. ${ }^{1}$ Recent findings showed that these gastroenteric abnormalities are partly related with changes of gut microbiota (GM) composition and its metabolic products. ${ }^{2}$ The GM is in turn influenced by diet, medication, the immune system, intestinal transit time, and other factors. ${ }^{3}$ To date, several studies have shown a significant difference between the GM of PD patients and healthy controls applying different study methods. ${ }^{4-8}$

At phylum level, for example, significant changes were observed in Firmicutes, Bacteroidetes and Proteobacteria. 4, 5, 9,10 Considering the taxonomic level of the family, several studies showed a reduction of Lachnospiraceae 11, 4, 5 and an increase in Enterobacteriaceae 5, 9 and Verrucomicrobiaceae, ${ }^{5,10,11,12}$ while at higher taxonomic resolution the data are discordant. ${ }^{10}$

Clearly, a basic understanding of compositional changes is needed to better recognize how the GM might impact onset and progression of PD, or in turn be influenced by the severity of the disease itself.

Another remarkable point of interest is to understand how medication impacts the gut microbiota. L-dopa (LD) represents the most potent dopamine replacement agent to treat PD. ${ }^{13}$ However, gut microorganisms are able to degrade LD even in the presence of decarboxylase inhibitors, thereby reducing its effectiveness. ${ }^{14-16}$ 
Previous researches discussed the treatment with LD as a possible influencing factor on GM. However, as far as we know, no study specifically addressed changes of the microbial community composition in PD related to LD medication in detail. ${ }^{4,7,11}$ Nonetheless, a recent study showed the important role of microbial metabolism in drug availability and degradation in the gut. ${ }^{14}$ These data suggest a complex interplay interaction between GM and disease progression, disease phenotype and antiparkinsonian medications, which still needs to be clarified.

The present study aims to investigate the faecal GM in a large cohort of PD patients, focusing on the potential impact of LD and, in particular, the intraduodenal infusion Levodopa-carbidopa intestinal gel (LCIG) in changing its composition and its metabolic products.

\section{METHODS}

This is a prospective investigational study (March 2017- May 2019) approved by the Institutional Review Boards and Human Subject Committees (Prot. PG/2017/17817).

A large cohort of 107 patients with diagnosed PD were recruited at the Neurology Department AO Brotzu (Cagliari, Italy) and at the Department of Neuroscience "Rita Levi Montalcini", University of Torino (Turin, Italy). Stool samples were collected following the same modalities in both the Cagliari and Turin centers. Samples from each patient were collected at home in a sterile refrigerated container and then delivered within 3 hours and stored at $-80^{\circ} \mathrm{C}$. All analyses were performed at the Laboratory of Microbiology and Virology at the Department of Biomedical Sciences, University of Cagliari. The samples collected in Turin were subsequently dry-ice shipped to the laboratory of Cagliari for the analysis.

We selected idiopathic PD patients diagnosed according to the UK Brain Bank criteria, excluding patients with atypical parkinsonism. The exclusion criteria were: the use of probiotic or antibiotic in the three months before enrollment; a primary gastrointestinal disease; the concomitant presence of an internal medicine, neurological, or unstable psychiatric illness or severe cognitive impairment. 
The PD patients were classified in different treatment groups: patients treated with LCIG (LCIGGroup) ( $n=38)$ and patients assuming LD (LD-Group) $(n=46)$. We also included a group of patients $(n=23)$ who had never taken drugs until the time of recruitment (Naïve-Group). (Tab.1)

All PD patients were stratified according to phenotype categories as tremor dominant (TD), with postural instability and gait difficulty (PIGD), and as dyskinetic patients (DP) in case of prominent dyskinesias (at least 2/4 in scores 4.32-4.33-4.34 in UPDRS IV) and/or onset of dyskinesias $<3$ aa from the start of therapy with L-DOPA. Furthermore, by elaborated a mean of previously defined turnpoints of advanced disease, ${ }^{19}$ we stratified patients according to disease duration in "early stage" (<3 years), "mid-stage" PD (3-13 years) and "andvanced-stage" (>13 years).

Our patients were evaluated by MDS-UPDRS III and IV and by Non-Motor Symptom Scale (NMSS). Diet was assessed by a validated questionnaire, ${ }^{17}$ physical activity, smoking status, constipation and coffee consumption were assessed through telephone interviews.

Microbiota analysis. DNA was extracted and purified from fecal samples and quantified by qPCR using the primers pair 5'-CCTACGGGNGGCWGCAG-3' (forward) and 5'GACTACHVGGGTATCTAATCC-3'(reverse), with genomic DNA from E. coli ATCC2592219 (see Supplementary Methods for details).

Statistical analysis. Pearson's chi squared tests were used to analyze differences in demographic and clinical characteristics. When cell frequency was $<5$ the Yates correction was applied.

The data analysis generated on the Miseq System was carried out using the BaseSpace 16S Metagenomics App (Illumina). Operational taxonomic units (OTUs) mapping to the Greengenes database (V.13.8) was performed using the Quantitative Insights into Microbial Ecology (QIIME) platform (V.1.8.0).

Alpha-diversity indices (ACE, Chao, Fisher, Shannon and Simpson) and beta-diversity analysis were performed on the Microbiome Analyst tool. ${ }^{18}$ The Linear discriminant analysis Effect Size (LEfSe)(http://huttenhower.sph.harvard.edu/galaxy/) was performed on Galaxy computational tool to identify bacterial taxa that were statistically different among the PD groups. The non-parametric 
factorial Kruskal-Wallis (KW) sum rank test and Wilcoxon rank-sum test were performed. The linear discriminant analysis (LDA) was used to estimate the effect size of each differentially abundant feature. The results were then corrected by the Benjamini and Hochberg false discovery rate (FDR) test for multiple comparisons.

To evaluate the effect of potential confounders on gut microbiota composition associated to dopaminergic medications, the General Linear Model (GLM) analysis was implemented, followed by Bonferroni correction for multiple comparisons using Statistical Package for the Social Sciences version (SPSS) 25.0 for Windows ${ }^{19}$ to test for confounding. Only bacteria that were found to be significant at the univariate level after FDR correction were considered. No normally distributed variables had been normalized using their logarithmic value before performing GLM.

The differences in microbiota composition among groups were adjusted for age, sex, BMI, constipation, diet, physical activity, coffee consumption, smoking status, phenotype, UPDRS-III and UPDRS-IV scales and disease duration covariates.

Metabolomics. Frozen feces from each participant were mixed in methanol solution and sonicated. After centrifugation, the supernatants were dried and derivatised with methoxyamine dissolved in pyridine. For GC-MS analysis, one microliter of derivatized sample was injected splitless into a 7890A gas chromatograph coupled with a 5975C Network mass spectrometer. (equipped with a fused silica capillary column (see Supplementary Methods for details). The correction for potential confounders on gut metabolome was implemented using GLM analysis followed by Bonferroni correction for multiple comparisons as described above for the microbiota analysis.

\section{RESULTS}

The extensive demographic and clinical characterization of PD patients is provided in Tab.1. Considering the two study groups (LD and LCIG Groups), they showed a relatively unbiased characterization for the common variables known to affect microbiota composition. Significant differences were displayed for sex, constipation, phenotype, UPDRS-III and UPDRS-IV scales and disease duration ( $\mathrm{p}$ values $<0.05)$. (Tab.1) 
A similar unbiased representation for the most important variables was observed in the comparisons between Naive-Group and the patients undergoing L-Dopa treatment (LD and LCIG Groups). However, significant differences were showed for sex, constipation, physical activity, phenotype, NMSS, UPDRS-III and UPDRS-IV scales and disease duration ( $p$ values $<0.05$ ). (Tab.1)

Considering COMT inhibitors (iCOMT), 6/46 patients in LD-Group (13\%) and 3/38 in LCIGGroup (8\%) were treated with these medicaments (Entacapone). Due to the small number of patients with iCOMT treatment it was not possible to assess the statistical significance of any difference between the LD group and the LCIG group. Consequently, iCOMT was not considered as a potential confounder in our analysis.

\section{Alpha/Beta diversity analysis}

No significant differences were found between the LD and LCIG groups in alpha diversity, as estimated throughout different indices ACE, Chao, Fisher, Shannon and Simpson $(p>0.05)$ (data not shown). On the contrary, the microbial beta-diversity analysis significantly differed between the two treatment groups $(\mathrm{R} 2=0.037, \mathrm{p}<0.001)$ (Fig. 1A $)$. The alpha-diversity differences were instead observed in the comparison between the LD and LCIG groups vs Naïve-Group with ACE, Chao and Fisher indexes (p-values $\leq$ 0.0001) (Fig. 2 A-C), except for Shannon and Simpson measuraments (pvalues $\geq 0.05$ ) (data not shown). Both treated groups showed a lower diversity compared to the Naïve untreated group. Inter-sample beta-diversity showed the separation between the LD and LCIG groups $v s$ the Naïve-Group $(\mathrm{R} 2=0.022, \mathrm{p}$-value $\leq 0.025$ and $\mathrm{R} 2=0.048$, $\mathrm{p}$ value $\leq 0.001$, respectively) (Fig. 1B-C).

\section{Microbial Composition in patients treated with LCIG, LD and Naïve-Group.}

The most relevant results obtained by LEfSE analysis revealed an increase of the Proteobacteria, Enterobacteriaceae, Escherichia and Klebsiella in the LCIG-Group compared to the LD-Group, as well as an increase in Parabacteroides and Serratia and a reduction of Firmicutes and Clostridiaceae and Lactobacillus in the LCIG- Group, compared to the LD-Group. (Fig.3C). However, after correction for confounders, the LCIG-Group showed a significant higher 
abundance of the Enterobacteriaceae family, Escherichia and Serratia genera compared to the LD-Group (Tab.2).

With regards to the comparison between the LD-Group and the Naïve-Group (Fig.3A), the LEfSE analysis showed the most important contribution in the Naïve-Group given by the Ruminococcaceae and Erysipelotrichaceae families, by Faecalibacterium, Roseburia and Pseudobutyrivibrio genera, and by Bacteroides Blautia and Lachnospira species. Moreover, Colinsella was increased in LD-Group. In the LEfSE comparison between the LCIG-Group and the Naïve-Group, Proteobacteria phylum and several species within Enterobacteriaceae family with Klebsiella, Escherichia and Enterobacter genera were more abundant in the LCIG- Group, while Firmicutes Ruminococcaceae, Faecalibacterium, Lachnospiraceae and Blautia were more abundant in the Naïve-Group. (Fig.3B)

After correction for multiple confounders, no significant difference was found in the multivariate analysis of the LD-Group vs Naïve-Group and the LCIG- vs Naive-Group.

\section{Metabolomics.}

A total of 90 metabolites were identified in the stool samples from PD patients by using GC-MS.

The results of the Orthogonal Partial Least Square-Discriminant Analysis (OPLS-DA) model obtained from the comparisons of LD and LCIG groups using the multivariate statistical analysis (MVA) are shown in Fig.4. OPLS-DA model quality parameters (R2y: 0.556; Q2: 0.326) and the respective permutation test (R2 intercept: 0.0, 0.333; Q2 intercept: 0.0, -0.161) displayed the statistical validity of the analysis performed using Partial Least Square-Discriminant Analysis (PLS-DA), indicating distinct metabolic profiles associated with the two different treatment groups.

With regards to the differences between LD and LCIG groups in the univariate analysis, 11 metabolites were significantly higher in the LCIG-Group: 2-amynobutyric acid, acetic acid, butyric acid, caffeic acid, coprostanol, coprostanone, fumaric acid, leucine, pentadecanoic acid, tetradecanoic acid, threonine. On the other hand, the LD-Group showed an increase in alanine, amynobutyric acid, aminovaleric acid, aspartic acid, galacturonic acid, glutamic acid, glutamine, hydroxycaproic acid, isoleucine, methionine, phenylalanine, proline, putrescine, thiamine, valine (Fig.S1).

This article is protected by copyright. All rights reserved 
Comparing the LD-Group and the Naïve-Group, 4-hydroxyphenylproprionic acid, alanine, cadaverine, glutamine, glycine, hydroxicaproic acid, methionine, phenylalanine, proline, threonine, valine were found significantly increased in LD-Group, while butyric acid, glyceric acid, hydrocinnamic acid, isoleucine, leucine, nicotinic acid and serine were significantly decreased (Fig.S2).

By contrast, the comparison between both LCIG and Naïve groups showed only fewer differences; i.e. 4-hydroxyphenylpropionic acid, cadaverine and tetradecanoid acid were significantly higher in LCIG-Group (Fig.S3).

When the same data were analyzed with the covariance analysis (ANCOVA) where age, sex, BMI, constipation, diet, physical activity, coffee consumption, smoking status, phenotype, UPDRS-III and UPDRS-IV scales and duration of disease were covariates, most of the metabolites differences between the two groups were lost. Only isoleucine, proline, threonine, pentadecanoic acid, tetradecanoic acid and caffeic acid maintained significant differences after the correction for confounder factors $(p$ values $<0.05$ ) in the comparison between LD and LCIG (Fig.S1). While alanine, glycine, hydroxicaproic acid and valine had maintained a significant increase in the LD-Group vs Naïve-Group (Fig.S2). No significant differences were observed in LCIG-Group vs Naïve Group ( $\mathrm{p}$ values $<0.05$ ).

\section{DISCUSSION}

The objectives of this study were to evaluate and compare the role of PD medicaments, specifically LD and its intestinal infusion therapy with LCIG, as modifying factors of the intestinal microbiota and of its metabolic profile. In order to better focus on the effect of medicaments, we also selected a subgroup of PD patients who had never undertaken any LD treatment (NaiveGroup) and compared their GM/metabolome profile to the LD-Group and LCIG-Group.

Our findings are novel in the field and of great importance. They have revealed that gut microbiota composition differs in PD patients in relation to the treatment with LD or LCIG. 
The potential modulation of microbiota by antiparkinsonian drugs has been previously evaluated by published papers: iCOMT were found to increase Enterobacteriaceae composition and to reduce Firmicutes, while carbidopa/LD was found to influence gut microbiota to the limit of statistical significance 5, 6, 11 However, these results were not consistent and a systematic metanalysis analyzed the effects of six PD drugs (levodopa/carbidopa, iCOMT, monoamine oxidase-B inhibitors) and found that only the intake of iCOMT had a significant effect on the overall microbiota composition. ${ }^{21}$

Concerning LD, some authors have recently found a significant influence on the relative abundances of some bacteria genera (such as Peptoniphilus, Fusicatenibacter, Enterococcus and others). ${ }^{22}$ These bacteria, capable of degrading defensive proteins provided by the host, might be able to induce irritable bowel syndrome and possess a variety of immune evasive functionalities and some of them (Enterococcus) were also described to convert LD to dopamine in the gut. Thus, LD might have a potential negative effect on local gut function, led by its interaction with gut microbiota.

Our findings showed that LD and LCIG had a lower alpha diversity compared to the Naïve untreated group when evaluating the overall gut microbiota community structure. In addition, all comparisons differ significantly in the microbial beta-diversity analysis. Suggesting that LD and LCIG treatments may impact the microbial community structure in PD.

These results were confirmed in our direct comparison between the LCIG-Group and the LDGroup where we found significant differences in bacteria taxa. LCIG was related with a significant higher abundance of the Enterobacteriaceae family, Escherichia and Serratia genera. The Enterobacteriaceae species, whose increase has already been reported in literature in treated PD patients 5,9 , are a large family of gram-negative facultative bacteria, residing in the gut at low levels in close proximity to the mucosal epithelium. Enterobacteriaceae are potential pathogens and their increase might be harmful due to the fermentation of lactic acid and the production of endotoxins. ${ }^{23}$ Consequently, our results suggest that LCIG might share the potential negative mechanisms of LD on gut microbiota with a more detrimental effect led by the higher intestinal biodisponibility of levodopa and by the overcome of gastric transit. 
Furthermore, the blooming of Enterobacteriaceae and Entorobacterial species has not been described in association with LD or LCIG, while it has been associated to gastrointestinal tract inflammation. As a result of inflammation, environmental and nutritional changes in the gut may confer a growth advantage to Enterobacteriaceae. Such an increase has been described in many conditions such as inflammatory bowel diseases (IBD), obesity, colorectal cancer, celiac disease, and PD itself. ${ }^{23}$ Therefore, we can speculate that the Enterobacteriaceae increase might be a consequence of higher gut inflammation led by LCIG. This effect might be linked to a local mechanical effect of the continuous infusion (up to 16 hours per day) through the percutaneous gastrojejunostomy, or by a potential detrimental effect of the infusion gel within LCIG.

Enterbobacteriaceae were previously found to be positively correlated with PD duration, postural instability 6,9 and iCOMT. ${ }^{6,11}$ In our cohort, only a few patients of the LD (6/46) and LCIG (3/38) groups were assuming iCOMT, consequently, as mentioned above, it was not possible to assess the statistical significance of any difference between these groups. Importantly, in our study the higher abundance of Enterobacteriaceae family, Escherichia and Serratia was significant also considering the correction for confounders (age, sex, BMI, constipation, diet, physical activity, coffee consumption, smoking status, phenotype, UPDRS-III and UPDRS-IV scales and disease duration). Hence, our data show a true correlation of higher Enterobacteriaceae, Escherichia and Serratia bacteria with LCIG treatment.

While we are aware that the microbiota composition might be influenced by multiple confounders, and that univariate correlations need to be cautiously interpreted, we believe that our univariate analysis shows interesting discussion topics. We found a reduction of Short Chain Fatty Acids (SCFAs) producing bacteria (Faecalibacterium, Roseburia, Blautia and Lachnospira spp) in both the LD-Group and LCIG-Group compared to Naïve-Group. SCFAs are signaling molecules produced from gut bacteria fermentation with anti-inflammatory and anti-oxidant properties ${ }^{24-26}$ and SCFAs-producing-bacteria deficiency has been frequently described in PD. ${ }^{4,5}$ This deficiency may result in some detrimental effects, including increased inflammation, increased risk of $\alpha$ Syn deposition in the gastro-intestinal tract, and microglial activation in the brain. ${ }^{27}$

On the contrary, our results suggest that the reduction of SCFAs producing bacteria might not be a distinctive trait of microbiota in PD but could be considered an effect led by LD treatment in its different route of administrations. This reduction might potentially contribute to the generation of a pro-inflammatory environment in the gut, with a higher effect in LCIG, where Blautia and 
Lachnospira spp were further reduced compared to the LD-Group. However, after adjusting for multiple confounders, these interesting findings were not confirmed in the multivariate analysis, also because of the small dimensions of the sample. Further studies with bigger patient samples are needed to confirm and to better explore this aspect.

The potential detrimental and pro-inflammatory effects mediated by antiparkinsonian medicaments with LD on GM also find confirmation in our direct evaluation of faecal metabolites in the metabolome analysis.

Our findings show that the altered composition of the GM matched metabolic changes, mostly affecting lipids, fatty acids, polyamines, metabolisms of amino acids, expanding previous metabolomic results. ${ }^{28}$

Interestingly, the most important differences observed between the two study groups, also confirmed after multivariate analysis, concerned the amino acid metabolism.

In particular, our results showed that LCIG-Group was characterized by an increase of threonine compared to the LD-Group. Some authors reported that threonine is involved in the mucin synthesis, with an association between increased levels and disintegration of the mucin layer. ${ }^{29}$ Furthermore, Enterobacteriaceae are capable of utilizing mucin-derived sugars. ${ }^{30}$ Thus the overgrowth of Enterobacteriaceae and the increase of threonine might be linked to an alteration of the intestinal permeability and might confirm its potential local and detrimental effect of LCIG on the gut environment described above.

Moreover, the interaction between microbiota and medicaments might potentially influence drugs absorption and metabolism. LCIG patients showed a reduction of isoleucine compared to LD, and since large neutral amino acids might compete with LD to cross the intestinal barrier ${ }^{29-31}$ it is possible that the reduction in the intestinal concentrations of this amino acid in the LCIG-Group may also be linked to an increased bioavailability of the drug.

Another interesting result regards the decreased levels of caffeic acid in the LD-group. This polyphenol has been associated with microbial action in the gut 32 and, similarly, to caffeine bioactive compound, eliciting anti-inflammatory and neuroprotective properties. We did not observe significant differences in coffee consumption between the LD and LCIG patients, and the multivariate analysis allowed us to exclude that altered level of caffeic acid was due to a 
difference in diet or caffeine consumption between the two groups, pointing to a solid effect on gut microbiota.

Moreover, other relevant drug-induced changes highlighted a decrease of anti-inflammatory metabolites (Butyric acid, acetic acid) in the LD-Group compared to the Naïve-Group, and an increase of pro-inflammatory metabolites (cadaverine and putrescine) in both the LD-Group and Naïve-Groups. It is also noteworthy that we found that both the LD and LCIG groups were associated to an increase in the 4-hydroxyphenylpropionic acid, an LD metabolite mediated by the intestinal flora ${ }^{32}$ supporting the possible existence of multiple metabolic pathways, as reported for gut bacterial tyrosine decarboxylases, ${ }^{33}$ in which LD itself might modulate the overgrowth of intestinal bacteria involved in its metabolism.

These results, however, were not confirmed in the multivariate analysis, and, as mentioned above, further studies are needed to better evaluate the potential interesting interactions of gut microbiota, antiparkinsonian drugs and metabolites in wider populations.

Our study presents limitations, shared with papers with similar methodology, due to some constraints in the research design. The analysis of gut microbiota was evaluated without jejunum and sigmoid mucosal biopsies, no re-test or longitudinal evaluations were provided (so we cannot exclude potential seasonal influence) and although our patient sample was larger compared to most published papers on this subject, our results need to be replicated in broader and homogeneous PD populations

However, our research also presents several significant strengths:

1. For the first time we evaluated the effects of different ways of LD amministration as LCIG;

2. The cohort of patients was larger compared to previous studies, including a subgroup of untreated naïve patients;

3. A multivariate analysis was performed considering many potential confounders; ${ }^{22,34}$

4. More significant and specific results were provided in comparison with previous reports on the levodopa/carbidopa effects on GM; ${ }^{11,34-36}$ 
5. The results are confirmed by the direct metabolomic analysis, already performed in our previous research, ${ }^{37-38}$ whereas most of the studies on this topic limited the analysis to an indirect functional prediction of the bacterial metabolic pathways. ${ }^{22,3,34}$.

\section{CONCLUSIONS}

Our results add an important piece of information that can help to substantiate a distinctive feature of gut microbiota in treated PD population.

As a major finding of our work, we provide evidence that LD, and mostly its intraudodenal injection (LCIG), might significantly influence GM composition, driving a metabolic dysregulation linked to a specific inflammatory gut microenviroment.

Even though our study evaluated multiple confounders and developed a multivariate analysis, our results have to be regarded as preliminary. Further research carried out in larger and multicentric cohorts and with a homogeneous PD population (with similar disease duration and disease severity) are necessary to better elucidate the relationships between gut microbiota and bacterial products, and to confirm the role of LD and LCIG on microbiota and its metabolites in PD patients.

Conflict of Interest: On behalf of all authors, the corresponding authors state that there is no conflict of interest.

Financial disclosure: This study was funded by Fondazione di Sardegna (grant n.20612/2018). None of the authors have received any funding from any institution, including personal relationships, interests, grants, employment, affiliations, patents, inventions, honoraria, consultancies, royalties, stock options/ownership, or expert testimony for the last 12 months. 
Aknowledgements: The authors kindly acknowledge Fondazione di Sardegna for the financial support (grant n.20612/2018), Associazione Sarda Malati Parkinson (ASAMPA) and Prof Carlo Anchisi for their assistance with subject recruitment.

Data availability statement: The authors confirm that the data supporting the findings of this study are available within the article. If needed, further data that support the findings of this study are available from the corresponding authors, [CG, SV], upon reasonable request.

\section{BIBLIOGRAPHY}

1) Cersosimo, M. G. \& Benarroch, E. E. Pathological correlates of gastrointestinal dysfunction in Parkinson's disease. Neurobiol. Dis. 46, 559-564 (2012)

2) Zhao, Y. \& Yu, Y.-B. Intestinal microbiota and chronic constipation. Springerplus 5,1130 (2016)

3) Pietrucci D, Cerroni R, Unida V, Farcomeni A, Pierantozzi M, Mercuri NB, Biocca S, Stefani A, Desideri A. Dysbiosis of GM in a selected population of Parkinson's patients. Parkinsonism Relat Disord. Aug; 65:124-130 (2019)

4) Keshavarzian, A. et al. Colonic bacterial composition in Parkinson's disease. Mov. Disord. 30, 1351-1360 (2015).

5) Unger, M. M. et al. Short chain fatty acids and gut microbiota differ between patients with Parkinson's disease and age-matched controls. Parkinsonism Relat. Disord. 32, 66-72 (2016)

6) Scheperjans, F. et al. Gut microbiota are related to Parkinson's disease and clinical phenotype. Mov. Disord. 30, 350-358 (2015)

7) Hopfner, F. et al. Gut microbiota in Parkinson disease in a northern German cohort. Brain Res. 1667, 41-45 (2017).

8) Barichella M, Severgnini M, Cilia R, Cassani E, Bolliri C, Caronni S, Ferri V et al. Unraveling gut microbiota in Parkinson's disease and atypical parkinsonism. Mov Disord. Mar;34(3):396-405(2019)

9) Li W, Wu X, Hu X et al Structural changes of gut microbiota in parkinson's disease and its correlation with clinical features. Sci. China Life Sci 60, 1223-1233, 2017

This article is protected by copyright. All rights reserved 
10) Bedarf, J.R. Hildebrand F, Coelho LP, et al. Functional implications of microbial and viral gut metagenome chagesinearly stage l-dopa-nä̈ve parkinson's disease patients. Genome Med 9, 39, 2017

11)Hill-Burns E.M., Debelius JW, Morton J, et al. Parkinson's disease and parkinson's disease medications have distinct signatures of the gut microbiome. Mov. Disord. 32, 739 749, 2017

12) Hasegawa $S$. Goto $S$, Tsuji $H$, et al. Intestinal dysbiosis and lowered serum lipopolysaccharide-binding protein in parkinson's disease. PLoS One 10, e0142164, 2015.

13) Salat, D. \& Tolosa, E. Levodopa in the treatment of Parkinson's disease: current status and new developments. J. Parkinsons Dis. 3, 255-269 (2013).

14) Van Kessel, S. P., Frye A.K., El-Gendy A.O., Castejon M., Keshavarzian A., Van DijK G., El Aidy S. Gut bacterial tyrosine decarboxylases restrict levels of levodopa in the treatment of Parkinson's disease. Nat. Commun. 10, 310 (2019);

15) Goldin, B. R., Peppercorn, M. A. \& Goldman, P. Contributions of host and intestinal microflora in the metabolism of L-dopa by the rat. J. Pharmacol. Exp. therapeutics 186, 160-166 (1973);

16) Maini Rekdal, V., Bess, E. N., Bisanz, J. E., Turnbaugh, P. J. \& Balskus, E. P. Discovery and inhibition of an interspecies gut bacterial pathway for Levodopa metabolism. Science 364.

17) Sofi F, Dinu M, Pagliai $G$ et al Validation of a literature-based adherence score to Mediterranean diet:the MEDI-LITE score, International Journal of Food Sciences and Nutrition, 2017 http://dx.doi.org/10.1080/09637486.2017.1287884

18) Dhariwal A, Chong J, Habib S, King IL, Agellon LB, Xia J. 2017. Microbiome Analyst: a web-based tool for comprehensive statistical, visual and meta-analysis of microbiome data. Nucleic Acids Res 45:180-8.

19) IBM Corp. IBM SPSS Statistics for Windows, Version 25.0. Armonk, NY: IBM Corp (Released 2017)

20) Berthold MR, Cebron N, Dill F. KNIME - The Konstanz Information Miner. ACM SIGKDD Explorations; 1-6 (2009) 
21) Hiroshi Nishiwaki, MD, Mikako Ito PhD, Tomohiro Ishida MS, Tomonari Hamaguchi MD, PhD, Tetsuya Maeda MD, PhD, et al. Meta-Analysis of Gut Dysbiosis in Parkinson's Disease Mov Disord Jun 18. doi: 10.1002/mds.28119. Online ahead of print (2020)

22) Weis S, Schwiertz A, Unger M et al. Effect of Parkinson's disease and related medications on the composition of the fecal bacterial microbiota. npj Parkinson's Disease 5: 28; https://doi.org/10.1038/s41531-019-0100-x (2019)

23) Zeng et al Mucosal Immunol 2017 Jan;10(1):18-26. doi: 10.1038/mi.2016.75. Epub Aug 24 (2016)

24) Bergman EN. Energy contributions of volatile fatty acids from the gastrointestinal tract in various species. Physiol. Rev. 70, 567-590, (1990)

25) Huuskonen J., Suuronen T, Nuutinen T, Kyrylenko S, Salminen A. Regulation of microglial inflammatory response by sodium butyrate and short-chain fatty acids. Br J Pharmacol 141, 874-880, (2004)

26) Tremlett H., Bauer KC, Appel-Cresswell S, Finlay BB, Waubant E The gut microbiome in human neurological disease: a review. Ann Neurol 81: 369-382, (2018)

27) Mulak A. Controversy on the Role of Short-Chain Fatty Acids in the Pathogenesis of Parkinson's Disease Movement Disorders, Vol. 33, No. 3, 2018,

28) Sun MF, Shen YQ. Dysbiosis of gut microbiota and microbial metabolites in Parkinson's disease. Ageing Res Rev; 45: 53-61 (2018)

29) Figura M, Kuśmierska K, Bucior E, Szlufik S, Koziorowski D, Jamrozik Z, Janik P. Serum amino acid profile in patients with Parkinson's disease. PLoS One 13: e0191670 (2018)

30) Lei S, Kamada N, Chen GY, Inohara N, Nunez G. Control of pathogens and pathobionts by the gut microbiota. Nat Immunol; 14:685-690 (2013)

31) Nutt JG, Woodward WR, Hammerstad JP, Carter JH, Anderson JL. The on-off phenomenon in Parkinson's disease: relation to levodopa absorption and transport. New England Journal of Medicine. 310(8):483-8 (1984)

32) Peppercorn MA and Goldman P. Caffeic Acid Metabolism by Bacteria of the Human Gastrointestinal Tract. Journal of Bacteriology Dec: 996-1000 (1971)

33) Van Kessel SP, Frye AK, El-Gendy AO, et al. Gut bacterial tyrosine decarboxylases restrict levels of levodopa in the treatment of Parkinson's disease. Nature Communications $10: 310(2019)$ 
34) Barichella M., Savergnini M., Cilia R., et al Unraveling Gut Microbiota in Parkinson's Disease and Atypical Parkinsonism Mov Disord. Mar;34(3):396-405. doi: 10.1002/mds.27581. Epub2018 Dec 21 (2019)

35) Zhao and Yu Intestinal microbiota and chronic constipation Springer Plus 5: 1130 DOI 10.1186 / s40064-016-2821-1 (2016)

36) Hannoun A., Ward D., Flahive J., Friedman J., et al Effect of Levodopa on gut microbiome in Parkinson's Disease (PD) (abstract). Mov. Disord; 34 (suppl 2) (2019)

37) Vascellari S, Palmas V, Melis M, Pisanu S, Cusano R, et al. Gut microbiota and metabolome alterations associated with Parkinson's disease. mSystems 5:e00561-20. https://doi.org/10.1128/mSystems.00561-20. (2020)

38) Accepted Manuscript Number: IJBIOMAC-D-20-05004RI 
Tab. 1 PD subjects characteristics

\begin{tabular}{|c|c|c|c|c|}
\hline Variable & $\begin{array}{l}\text { PD patients } \\
\text { (Naive }=23 \text { ) }\end{array}$ & $\begin{array}{l}\text { PD patients } \\
(L D=46)\end{array}$ & $\begin{array}{l}\text { PD patients } \\
(\mathrm{LClG}=38)\end{array}$ & $p$ value \\
\hline Age, mean \pm SD & $67,57 \pm 9,37$ & $69,85 \pm 11,26$ & $72,38 \pm 9,36$ & $\begin{array}{l}0.473(\mathrm{~N}-\mathrm{L}) \\
0.670(\mathrm{~N}-\mathrm{D}) \\
0.224(\mathrm{D}-\mathrm{L})\end{array}$ \\
\hline BMI, mean \pm SD & $25,20 \pm 3,25$ & $26,79 \pm 4,27$ & $24,15 \pm 4,37$ & $\begin{array}{l}0.187(\mathrm{~N}-\mathrm{L}) \\
0.366(\mathrm{~N}-\mathrm{D}) \\
0.764(\mathrm{D}-\mathrm{L})\end{array}$ \\
\hline \multicolumn{5}{|l|}{ Sex, n (\%) } \\
\hline $\begin{array}{l}\text { Male } \\
\text { Female }\end{array}$ & $\begin{array}{l}13(56.52) \\
10(43.47)\end{array}$ & $\begin{array}{l}36(78.30) \\
10(21.70)\end{array}$ & $\begin{array}{l}20(52.60) \\
18(47.40)\end{array}$ & $\begin{array}{l}0.037(\mathrm{~N}-\mathrm{L}) \\
0.906(\mathrm{~N}-\mathrm{D}) \\
0.013(\mathrm{D}-\mathrm{L})\end{array}$ \\
\hline \multicolumn{5}{|l|}{ Constipation, n (\%) } \\
\hline Yes & $5(21.74)$ & $19(44.20)$ & $27(71.10)$ & $0.071(\mathrm{~N}-\mathrm{L})$ \\
\hline No & $18(78.26)$ & $24(55.80)$ & $11(28.90)$ & $0.000(N-D)$ \\
\hline Missing & 0 & 3 & 0 & $0.015(D-L)$ \\
\hline \multicolumn{5}{|l|}{ Coffee consumption, $\mathrm{n}(\%)$} \\
\hline $\begin{array}{l}\text { Yes } \\
\text { No }\end{array}$ & $\begin{array}{l}17(73.91) \\
6(26.08)\end{array}$ & $\begin{array}{l}29(67.40) \\
14(32.60)\end{array}$ & $\begin{array}{l}22(61.10) \\
14(38.90)\end{array}$ & $\begin{array}{l}0.586(\mathrm{~N}-\mathrm{L}) \\
0.311(\mathrm{~N}-\mathrm{D}) \\
0.558(\mathrm{D}-\mathrm{L})\end{array}$ \\
\hline \multicolumn{5}{|l|}{ Smoking status, $\mathrm{n}(\%)$} \\
\hline Yes & $1(4.35)$ & $4(9.30)$ & $0(0)$ & $0.469(\mathrm{~N}-\mathrm{L})$ \\
\hline No & $22(95.65)$ & $39(90.70)$ & $34(100)$ & $0.220(\mathrm{~N}-\mathrm{D})$ \\
\hline Missing & 0 & 3 & 4 & $0.068(D-L)$ \\
\hline \multicolumn{5}{|l|}{ Diet, n (\%) } \\
\hline Omnivorous & $17(94.40)$ & 26(96.30) & $33(97.10)$ & $0.768(\mathrm{~N}-\mathrm{L})$ \\
\hline Gluten free & $0(0)$ & $0(0)$ & $1(2.90)$ & $0.297(\mathrm{~N}-\mathrm{D})$ \\
\hline No Milk and milk derivatives & $1(5.60)$ & $1(3.70)$ & $0(100)$ & $0.358(D-L)$ \\
\hline Missing & 5 & 19 & 4 & \\
\hline \multicolumn{5}{|l|}{ Whole food, n (\%) } \\
\hline Yes & $8(44.40)$ & $11(40.70)$ & $7(20.60)$ & $0.805(\mathrm{~N}-\mathrm{L})$ \\
\hline No & $10(55.60)$ & $16(59.30)$ & $27(79.40)$ & $0.071(N-D)$ \\
\hline Missing & 5 & 19 & 4 & $0.087(D-L)$ \\
\hline \multicolumn{5}{|l|}{ Oil consumption, $\mathrm{n}(\%)$} \\
\hline $8 g$ & $7(38.89)$ & $9(33.34)$ & $6(17.65)$ & $0.988(N-L)$ \\
\hline $16 g$ & $5(27.78)$ & $9(33.34)$ & $8(23.53)$ & $0.612(N-D)$ \\
\hline $24 g$ & $1(5.56)$ & $2(7.41)$ & $3(8.82)$ & $0.389(D-L)$ \\
\hline $32 \mathrm{~g}$ & $1(5.56)$ & $2(7.41)$ & $4(11.76)$ & \\
\hline $40 \mathrm{~g}$ & $1(5.56)$ & $1(3.70)$ & $6(17.65)$ & \\
\hline
\end{tabular}




\begin{tabular}{|c|c|c|c|c|}
\hline $48 g$ & $1(5.56)$ & $1(3.70)$ & $5(14.71)$ & \\
\hline $56 g$ & $1(5.56)$ & $2(7.41)$ & $1(2.94)$ & \\
\hline $64 \mathrm{~g}$ & $1(5.56)$ & $1(3.70)$ & $1(2.94)$ & \\
\hline Missing & 5 & 19 & 4 & \\
\hline \multicolumn{5}{|l|}{ Supplements, n (\%) } \\
\hline Yes & $2(11.01)$ & $0(0)$ & $3(8.80)$ & $0.082(\mathrm{~N}-\mathrm{L})$ \\
\hline No & 16 (88.90) & $26(100)$ & 31 (91.2) & $0.790(\mathrm{~N}-\mathrm{D})$ \\
\hline Missing & 5 & 20 & 4 & $0.120(D-L)$ \\
\hline \multicolumn{5}{|c|}{ Balanced nutrition, n (\%) } \\
\hline Adequate & 12(66.70) & $21(77.80)$ & $22(64.70)$ & $0.409(N-L)$ \\
\hline Not adequate & $6(33.3)$ & $6(22.20)$ & 12(35.30) & $0.888(N-D)$ \\
\hline Missing & 5 & 19 & 4 & $0.266(D-L)$ \\
\hline \multicolumn{5}{|c|}{ Physical activity, n(\%) } \\
\hline No activity & $5(22.70)$ & 13(30.20) & $18(50.00)$ & $0.368(N-L)$ \\
\hline Moderate & $7(31.80)$ & $18(41.90)$ & $15(41.70)$ & $0.000(N-D)$ \\
\hline Regular & $10(45.50)$ & $12(27.90)$ & $3(8.30)$ & $0.052(D-L)$ \\
\hline Missing & 1 & 3 & 2 & \\
\hline \multicolumn{5}{|l|}{ Phenotype, n (\%) } \\
\hline Tremor-dominant & $19(79.20)$ & $20(43.50)$ & $4(10.05)$ & $0.005(\mathrm{~N}-\mathrm{L})$ \\
\hline Akinetic-rigid & $4(16.70)$ & $18(39.10)$ & $15(39.50)$ & $0.000(N-D)$ \\
\hline Dyskinetic & $0(0)$ & $8(17.40)$ & $19(50.45)$ & $0.001(D-L)$ \\
\hline \multicolumn{5}{|l|}{ NMSS, $\mathrm{n}(\%)$} \\
\hline $0-39$ & 20(90.90) & $2(40.00)$ & $4(14.30)$ & $0.008(\mathrm{~N}-\mathrm{L})$ \\
\hline $40-80$ & $2(9.10)$ & $3(60.00)$ & $14(50.00)$ & $0.000(N-D)$ \\
\hline$>80$ & $0(0)$ & $0(0)$ & 10 (35.70) & $0.182(\mathrm{D}-\mathrm{L})$ \\
\hline Missing & 1 & 41 & 10 & \\
\hline \multicolumn{5}{|l|}{ UPDRS-III, n(\%) } \\
\hline Mild (0-32) & 20(90.90) & $21(65.60)$ & $12(36.40)$ & $0.077(\mathrm{~N}-\mathrm{L})$ \\
\hline Moderate (33-58) & $2(9.10)$ & $7(21.90)$ & $16(48.50)$ & $0.000(N-D)$ \\
\hline Severe $(>58)$ & $0(0)$ & $4(12.50)$ & $5(15.20)$ & $0.048(D-L)$ \\
\hline Missing & 1 & 14 & 5 & \\
\hline \multicolumn{5}{|l|}{ UPDRS-IV, n(\%) } \\
\hline Mild (0-4) & $22(100)$ & $20(64.50)$ & $12(32.40)$ & $0.007(\mathrm{~N}-\mathrm{L})$ \\
\hline Moderate (5-12) & $0(0)$ & $9(29.00)$ & $21(56.80)$ & $0.000(N-D)$ \\
\hline Severe $(>12)$ & $0(0)$ & $2(6.50)$ & $4(10.80)$ & $0.030(D-L)$ \\
\hline Missing & 1 & 15 & 1 & \\
\hline \multicolumn{5}{|c|}{ Duration of disease, $n(\%)$} \\
\hline $0-3$ years & $17(73.90)$ & $11(29.70)$ & $0(0)$ & $0.004(\mathrm{~N}-\mathrm{L})$ \\
\hline 4-13 years & $5(21.70)$ & $21(56.80)$ & $13(34.20)$ & $0.000(N-D)$ \\
\hline$>13$ years & $1(4.30)$ & $5(13.5)$ & $25(65.80)$ & $0.000(D-L)$ \\
\hline Missing & 0 & 9 & 0 & \\
\hline ICOMT, n (\%) & 0 & $6(13 \%)$ & $3(8 \%)$ & 0.447 (D-L) \\
\hline
\end{tabular}


Tab.1 Characteristics associated to Parkinson subjects. Values in the groups were presented as: $\mathrm{M}=$ Mean, $\mathrm{SD}=$ Standard deviation, $\mathrm{n}=$ number, $\%=$ Percentage. Abbreviation: BMI= Body Mass Index; $\mathrm{N}=$ Naive; L= LD; D= LCIG, ICOMT=Inhibitors of Catechol-O-Methyltransferase (COMT); $p$ values were calculated with Student t-test for Age and BMI. For the other variables Chi Square test was performed. 
Tab.2.-Multivariate Analysis differences of gut microbiota in LCIG-Group vs LD-Group

\begin{tabular}{|c|c|c|c|c|c|c|}
\hline & Family & Genus & Species & Type & & p value* \\
\hline \multirow[t]{3}{*}{ Proteobacteria } & Enterobacteriaceae & & & 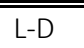 & $\bar{\downarrow} \downarrow$ & 0.010 \\
\hline & & Escherichia & & L-D & $\downarrow$ & 0.035 \\
\hline & & Serratia & & L-D & $\downarrow$ & 0.002 \\
\hline
\end{tabular}

Tab.2 Generalized Linear Model (GLM) followed by Bonferroni correction for multiple comparisons in Statistical Package for the Social Sciences Version (SPSS) 25.0 for Windows. The differences of microbiota composition between PD patients treated with LD (L) and LCIG (D) were adjusted for sex, age, BMI, physical activity, constipation, phenotype, disease duration, coffee consumption, smoking status, diet, oil consumption, supplement, balanced nutrition, NMSS UPDR-III and UPDRSIV scales covariates. Bacteria with a relative abundance $\geq 0.1 \%$ in at least $25 \%$ of the population of one of the three study groups were considered; Bonferroni corrected $\mathrm{p}$ values ( $\mathrm{p}$-value*): $<0.05$. 
A

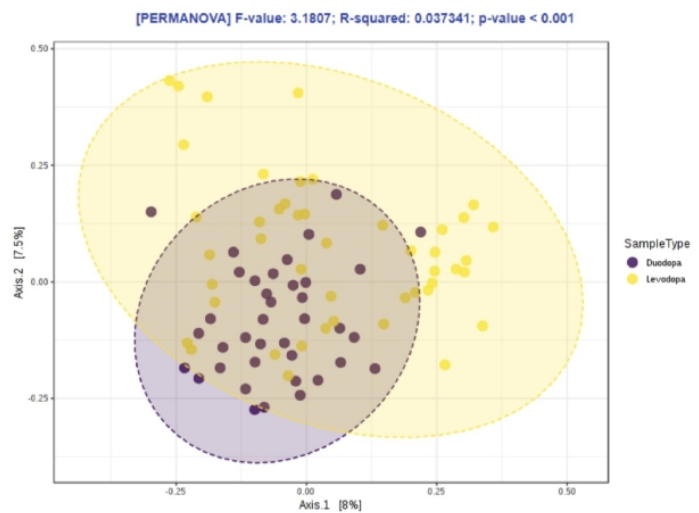

B

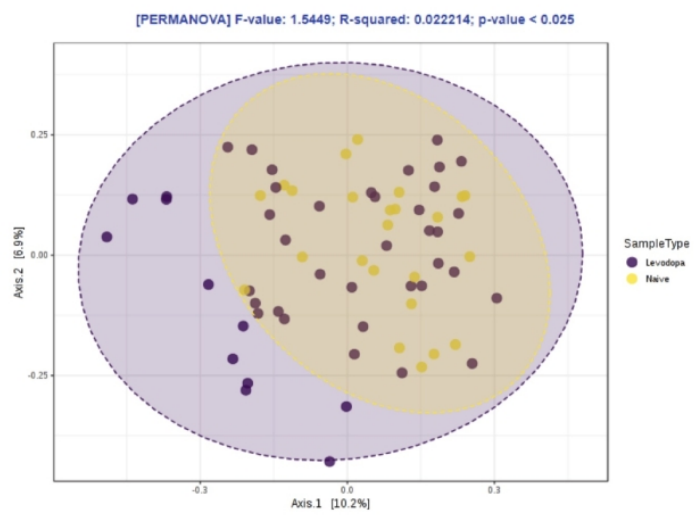

C

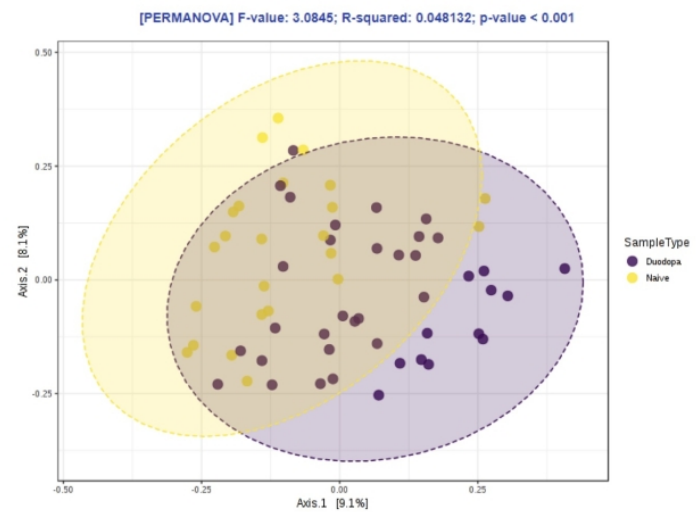

ene_14644_f1.jpg

This article is protected by copyright. All rights reserved 
A

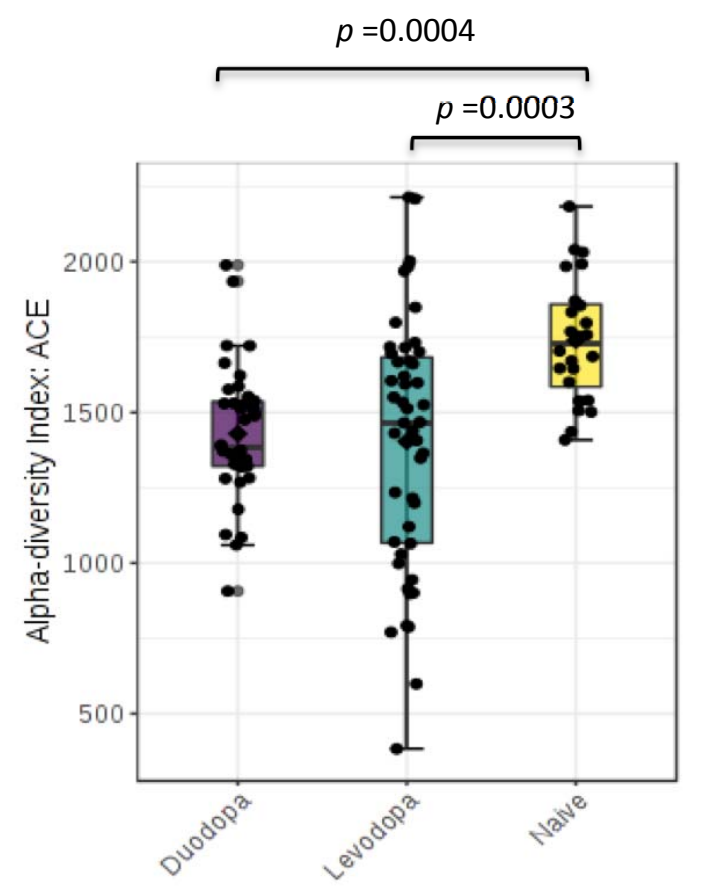

B

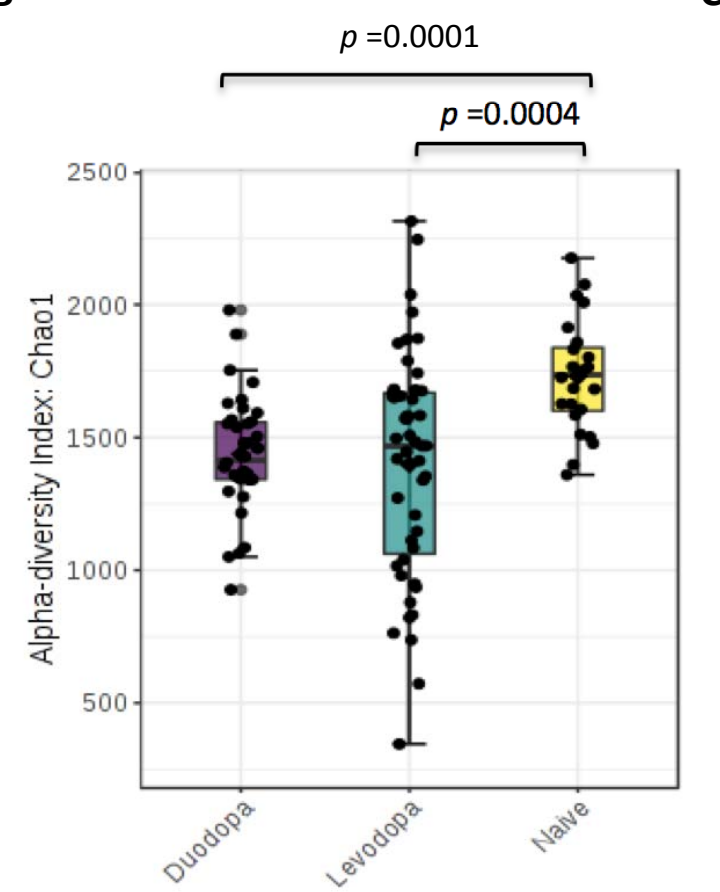

C

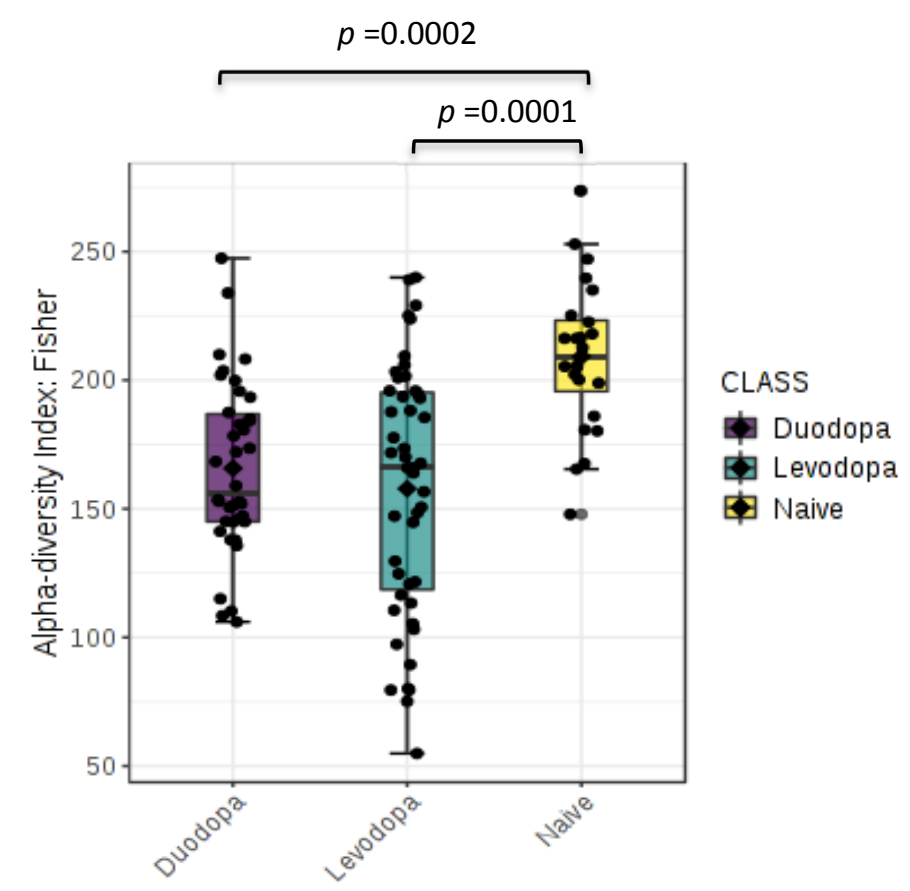




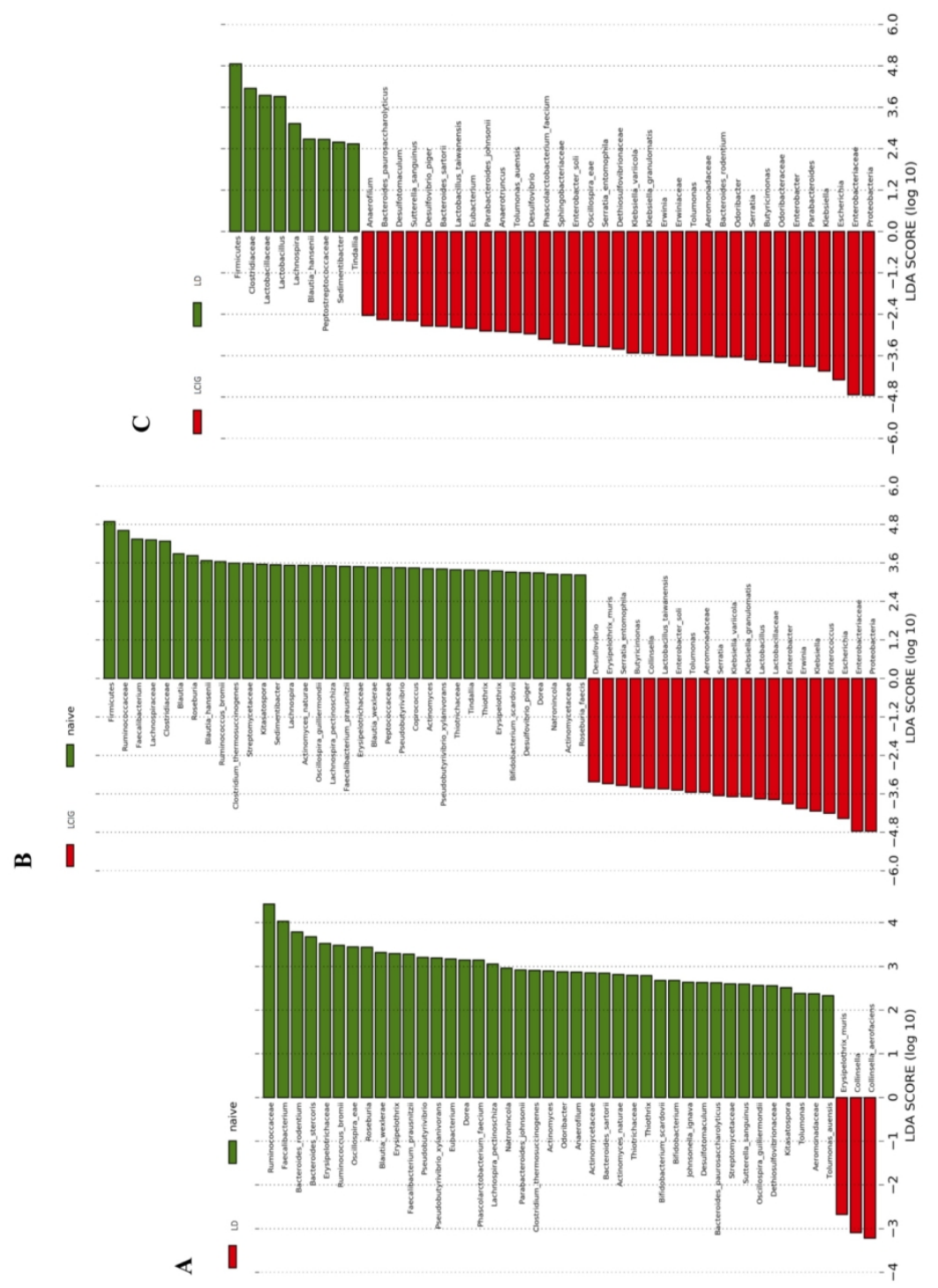

ene_14644_f3.jpg

This article is protected by copyright. All rights reserved 
a

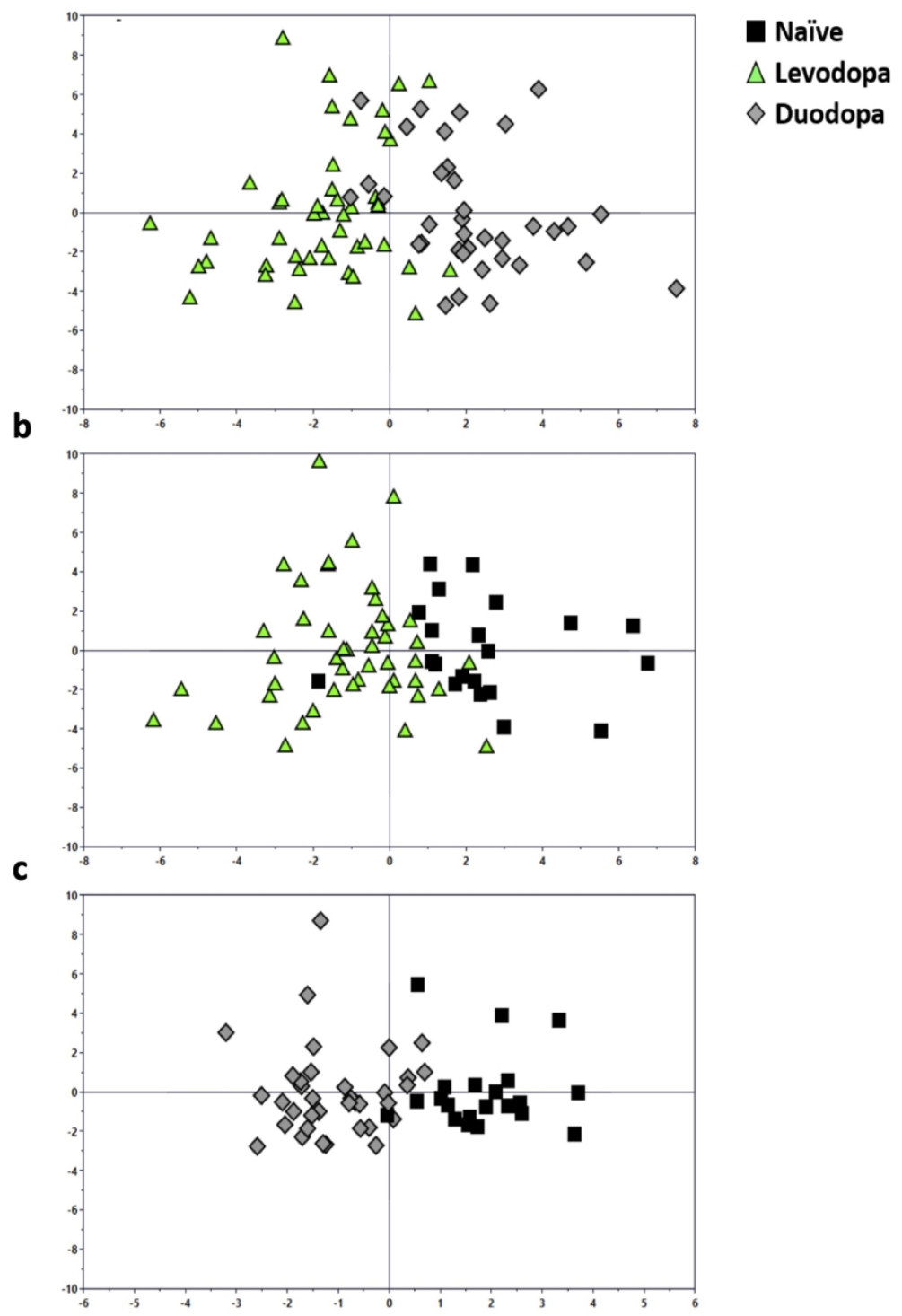

ene_14644_f4.jpg

This article is protected by copyright. All rights reserved 